\title{
Vitalfärbung an den Pollenmutterzellen von Lilium speciosum
}

Von

\author{
G. Yamaha \\ Aus dem Botanischen Institut der Tokyo-Universität für \\ Literatur und Wissenschaft
}

Eingegangen am 9. August $193^{8}$

Die in Teilung begriffenen Pollenmutterzellen zeichnen sich durch ungemein hohe Empfindlichkeit gegenüber verschiedenen Ionen aus (YAMAHA u. Ishi 1932, Yamaha 1937 usw.). Der Umstand läßt ohne Weiteres auf dis hohe Permeabilität des Protoplasmas der betreffenden Zellen auch für Farbstoffe schließen. Vitalfärbung der Protoplasten wurde schon früher von uns bei den Pollenmutterzellen einiger Pflanzen erzielt, und zwar mit den Farbstoffen aus Sulfophtaleinen (Bromphenolblau, Bromkresolgrün, Bromkresolpurpur), womit sich Protoplasma sonst schwer vital färben läßt. (YамAнa u. Ishil 1932, 1933, Yamaha 1937 usw.)

Im Zusammenhang mit den Untersuchungen über die elektrische Struktur (Ladungsverhältnisse, Potentialdifferenz usw.) des Protoplasmas in der sich teilenden Pollenmutterzelle wurden eine Reihe von Färbungsversuchen mit demselben Material vorgenommen, um über die Vitalfärbbarkeit verschiedener Strukturelemente der Protoplasten klar zu werden. Als Versuchsobjekt dienten Pollenmutterzellen von Lilium speciosum, und zwar wurden nur solche herangezogen, die sich gerade in der ersten Meta- oder Anaphase der Meiosis befanden. Färbung wurde in zweierlei Weisen ausgeführt:

zu 1. wurde ein möglichst kleines Tröpfchen von Farbstofflösungen (Konzentration 0,1 oder 0,05 proz. bei sauren Farben, 0,01 oder 0,005 proz. bei basischen Farben) dem aus der Anthere herausgepressten Schleim mitsamt den Pollenmutterzellen auf dem hohl geschliffenen Objektträger zugesetzt. Nach dem Auflegen des Deckglases dauerte die Beobachtung unter Mikroskop 5-20 Minuten, wobei ZEIßsches Komp. Okular $\times 20$ und apochr. Objektiv $\times 40 \mathrm{zur}$ Anwendung kamen. Diese Reihe von Färbungsversuchen könnten aber nur als Orientierungsversuche gelten. In diesem Fall wird die Ionenkonzentration des Antherenschleimes durch den Zusatz der Farbstofflösung verändert, so daß die Pollenmutterzellen dadurch ohne Zweifel in abnormen Zustand versetzt werden. Ionenkonzentration des Beobachtungsmediums bleibt hier völlig unbekannt (vgl. YAMAHA 1937b). Vitalfärbung unter bestimmten Bedingungen erscheint nun dringend nötig. Die zweite Reihe von Färbungsversuchen entsprechen diesem Erfordernisse. 
zu 2. mußte die Vitalfärbung in dem für die Pollenmutterzellen günstigsten Beobachtungsmedium erfolgen. Solch ein Medium fand ich nach Ausprobieren neben Paraffinöl (wassergesättigt) in 0,050,2 GM Phosphatpuffer (ein Gemisch von $\mathrm{KH}_{2} \mathrm{PO}_{4}$ und $\mathrm{K}_{2} \mathrm{HPO}_{4}$ ) von demselben $\mathrm{pH}$ wie Antherenschleim ( $\mathrm{pH}$ 6.2, vgl. YamaHa 1938). Vitalfärbung in Paraffinöl ist bei diesem Objekt nicht gelungen, weil sie hier so langsam zu erfolgen scheint, daß die Pollenmutterzellen immer geschädigt erscheinen, bevor sie vital gefärbt werden. Ausserdem haben sich nur eine geringe Anzahl von Farbstoffen als paraffinöllöslich erwiesen (vgl. YAMAHA 1937b). So kommt als Lösungsmittel von Farbstoffen im Allgemeinen 0,1 GM Phosphatpuffer von $\mathrm{pH} 5,0-8,0$ zur Anwendung. Daneben wurden Farbstoffe in zweimal destilliertem Wasser oder in $0,1 \mathrm{n} \mathrm{KCl}$ von $\mathrm{pH} \mathrm{5,0-8,0}$ gelöst. pH-Bestimmung erfolgte mittels Antimon- oder Glaselektrode gleich vor der Färbung.

Folgende 58 Arten der Farbstoffe wurden geprüft:

I. Saure Farbstoffe (einschließlich amphoterer Farbstoffe)

(1) Nitrofarbstoffe. Aurantia

(2) Monazofarbstoffe. Orange G, Methylorange, Tropaeolin OO, Methylrot

(3) Diazofarbstoffe. Kongorot, Kongorubin, Trypanrot, Trypanblau

(4) Triphenylmethanfarbstoffe. Anilinblau, Ketonblau $4 \mathrm{BN}$, Lichtgrün SF, Rubin S, Methylblau, Wasserblau, Isaminblau, Zyanol extra, Korallin

(5) Xanthenfarbstoffe. Sulphorhodamin, Rhodamin B, Eosin, Erythrosin, Bengalrosa

(6) Indigofarbstoffe. Indigkarmin

(7) Sulfophtaleine. Chlorphenolrot, Bromkresolpurpur, Bromphenolblau, Bromkresolgrün

(8) Natürliche Farbstoffe. Hämatoxylin

II. Basische Farbstoffe

(1) Monazofarbstoffe. Chrysoidin, Chrysoidin R, Janusgrün

(2) Diazofarbstoffe. Bismarckbraun

(3) Triphenylmethanfarbstoffe. Malachitgrïn, Dahlia, Fuchsin, Methylviolett, Pyoktanin, Gentianaviolett, Methylgrün, Jodgrün, Nachtblau, Viktoriablau 4R, Viktoriablau B

(4) Xanthenfarbstoffe. Pyronin

(5) Indaminfarbstoffe. Toluylenblau

(6) Azinfarbstoffe. Neutralrot, Safranin T, Phenosafranin, Baslerblau, Mauvein, Magdalarot

(7) Oxazinfarbstoffe. Brillantkresylblau, Prune pure, Nilblau

(8) Thiazinfarbstoffe. Thionin, Methylenblau, Toluidinblau

Als Vitalitätskriterium galt neben dem mikroskopischen Aussehen der Zellen Brownsche Molekularbewegung im Zytoplasma. Der Verlauf der Karyokinese in der vitalgefärbten Zelle konnte leider nicht verfolgt werden. 


\section{Vitalmembranfärbung}

Schöne Membranfärbung ohne Plasmamitfärbung kann man mit folgenden Farbstoffen beobachten:

Kongorot, Trypanblau, Lichtgrün SF, Methylblau, Wasserblau, Zyanol extra, Anilinblau, Indigkarmin.

Bei Lichtgrün SF und Trypanblau wurde vereinzelt auch schwache Plasmamitfärbung bemerkt. Weiterhin wird bei folgenden Farbstoffen vitale Membranfärbung von der diffusen Plasmafärbung begleitet:

Methylorange, Kongorubin, Isaminblau, Trypanrot, Malachitgrün, Bismarckbraun, Bromkresolgrün, Bromphenolblau, Bromkresolpurpur.

Grüne metachromatische Färbung der Zellmembran tritt bei Zyanol extra und Indigkarmin auf. Wasserblau hat sich als ziemlich giftig erwiesen, da die Pollenmutterzellen bald nach der Membranfärbung eine deutliche Koagulation des Protoplasmas erfahren.

Es verdient eine besondere Beachtung, daß es im Allgemeinen zu sauren Farbstoffen gehören, welche die Zellmembran der Pollenmutterzellen vital färben. Diese sauren Farbstoffe, die den Azo-, Triphenylmethan- und Indigofarbstoffen angehören, sind nach eigenen Kataphorese-Versuchen ${ }^{1)}$ in wässriger Lösung ohne Ausnahme elektrisch negativ geladen. Daß die negativen Farbstoffe Zellmembran anfärben können, was nach früheren Angaben (vgl. z.B. CzAJA 1936 usw.) unwahrscheinlich erscheint, läßt sich im übrigen bei den sich teilenden Pollenmutterzellen mit Leichtigkeit erwarten, wenn man berücksichtigt, daß die Oberfläche der Zellmembran der letzteren der umgebenden Flüssigkeit $(0,1 \mathrm{n} \mathrm{KCl)}$ gegenüber elektrisch positiv geladen ist (YAMAHA 1938b), und weiter daß die betreffende Zellwand hier im Gegensatz zu gewöhnlichen pflanzlichen Zellen weder Zellulose noch Pektinstoffe enthält, sondern aus einem chemisch noch unbekannten Stoff besteht, der sich nach mikrochemischen Reaktionen wie Kallose (MANGIN 1892, 1910) verhält (Yamaha 1920). Kallose färbt sich angeblich mit sauren Farbstoffen (Kongorot, Korallin). Mit Korallin (Rosolsäure, gelöst in 0,1 GM Phosphatpuffer von $\mathrm{pH}$ 9,0) konnte ich aber vitale Membranfärbung bei den Pollenmutterzellen nicht erzielen. Dabei bemerkt man schwache diffuse Plasmafärbung (postmortal?).

$\mathrm{Zu}$ dieser Regel machen Malachitgrün und Bismarckbraun Ausnahmen, welche beide chemischer Konstitution nach offenbar zu

1) Dabei kam eine Doppel-U-Röhre, nicht aber ein Paar KCl-Agar-Elektroden zur Anwendung. 
basischen Farbstoffen gehören, obwohl nach MCCLung und CoNN (1937) der erstere Farbstoff als ,schwach basisch“, und der letztere als ,schwach sauer" bezeichnet ist (vgl. weiter ConN 1936, S. 97). Die beiden geprüften Farbstoffe stammen aus der Firma GrüBLER, Leipzig und nach eigenen Kataphorese-Versuchen sind sie in wässriger Lösung deutlich elektrisch positiv geladen. Die Ursache für die vitale Membranfärbung mit diesen beiden Farbstoffen ist vorderhand nicht einzusehen.

Mit einer nicht geringen Anzahl von sauren Farbstoffen, besonders aus der Gruppe von Nitro- und Xanthenfarbstoffen, bemerkt man vitale diffuse Plasmafärbung, wobei sich die Zellmembran nicht im geringsten färben läßt (s. unten). Schließlich erzielt man mit den übrigen sauren Farbstoffen bei den Pollenmutterzellen gar keine Art der Vitalfärbung (Methylrot, Rubin S, Korallin, Sulforhodamin, Hämatoxylin und Chlorphenolrot).

Bisher ist häufig hervorgehoben, daß die Zellmembran der lebenden pflanzlichen Zellen sehr schwierig sich färben läßt. Einige Autoren bemerkten Membranfärbung mit sauren sowie basischen Farbstoffen nur bei verletzten Zellen oder erst nach dem Abtöten der Zellen (s. BECKER 1936, S. 453). Bei den Pollenmutterzellen beobachtet man jedoch Membranfärbung mit sauren Farbstoffen im dem mikroskopischen Aussehen nach völlig gesunden Zustand der Zellen, was meines Wissens auf eine besondere physikochemische (elektrische?) Beschaffenheit der Zellwand der Pollenmutterzellen zurückzuführen ist.

Bemerkenswert sei noch, daß bei der Vitalfärbung mit sauren (amphoteren) Farbstoffen die Zellmembran ohne Ausnahme die Farbe des Farbsalzes (alkalische Farbe) annimmt, während mit basischen Farbstoffen sie im Allgemeinen die der Farbbase (auch alkalische Farbe) zeigt. (CzAJA 1936). Wir finden also auch mit sauren Farbstoffen ,alkalischen Membraneffekt" wieder. So z.B. färbt sich die Zellmembran mit Methylorange gelb, Kongorot rot, Bromphenolblau schwach purpur, Bromkresolgrün blau. Mit Bromkresolpurpur bemerkt man schöne blaue Färbung der neugebildeten Scheidewand sowie der inneren Schicht der Membran der Pollenmutterzellen.

\section{Vitalplasmafärbung}

Die reine, d.h. nicht von Membranfärbung begleitete, diffuse Färbung des Zytoplasmas der Pollenmutterzellen beobachtet man bei folgenden Farbstoffen.

Saure Farbstoffe: Aurantia, Orange G, Tropaeolin OO, Ketonblau, Rhodamin B, Eosin, Erythrosin, Bengalrosa. 
Basische Farbstoffe: Chrysoidin, Chrysoidin R, Fuchsin, Dahlia, Methylviolett, Gentianaviolett, Pyoktanin, Jodgrün, Nachtblau, Viktoriablau B u. 4R, Safranin, Phenosafranin, Magdalarot, Kresylblau, Nilblau, Thionin.

Besonders schön tritt die diffuse Färbung des Zytoplasmas bei Aurantia, Tropaeolin OO, Pyoktanin und Magdalarot hervor. Plasmafärbung kommt nur schwach vor bei Bengalrosa, Gentianaviolett, Nachtblau, Viktoriablau B u. 4R und Nilblau. Weiterhin kann man mit Methylorange, Kongorubin, Trypanrot, Bismarckbraun und Malachitgrün diffuse Plasmafärbung mit gleichzeitiger Membranfärbung erzielen (s. oben). Ähnliche Verhältnisse findet man auch bei den Sulfophtaleinen. Auffallend ist nun zu bemerken, daß mit Sulfophtaleinen die Granulafärbung des Zytoplasmas zutage tritt, was insbesondere im verletzten Zustand der Protoplasten ausgeprägt erscheint. Dabei färben sich die Granula gelb (saure Farbe), das Hyaloplasma nimmt dagegen Salzfarbe (alkalische Farbe) an. Es scheint sich hier um eine Art "saurer Suspendierungseffekte" nach CzAJA (1934) zu handeln, da die dispersen Phasen des Protoplasmas elektrisch negativ geladen sind (YAmAHA 1937). Ähnliche Deutung hat SPEK (1937) früher bei der Vitalfärbung tierischer Eier geäußert. Dieser Befund sei für die pH-Bestimmung des Protoplasmas mittels Vitalfärbung von höchster Bedeutung, da die dispersen Phasen des Protoplasmas, die die betreffenden sauren Suspendierungseffekt hervorrufen, bis zu den submikroskopischen Größen hinunter gehen können, wobei sich das Hyaloplasma (Dispersionsmittel) und die dispersen Phasen (Submikronen) voneinander mikroskopisch nicht mehr unterscheiden lassen. Durch die Veränderung der Ionenkonzentration ( $\mathrm{pH}$ sowie Salzkonzentration) der umgebenden Flüssigkeit (Antherenschleim oder Beobachtungsmedium) kann man den Dispersitätsgrad sowohl des Zytoplasmas als auch des Karyoplasmas, von dem optisch leeren (amikroskopischen) Zustand bis in den völlig koagulierten, in jeder möglichen Stufe willkürlich verändern, so daß der angebliche Unterschied einerseits, zwischen dem Hyaloplasma und den Mikrosomen, und andererseits zwischen dem Kernsaft und den Karyotingranulis, nur scheinbar erscheint. Auf diese Frage möchte ich an einer anderen Stelle näher eingehen. Aus demselben Grund läßt sich jedenfalls auch zwischen der Granula- und diffusen Färbung keine scharfe Grenzlinie ziehen. Im Gegensatz zum tierischen Objekt liegen bisher nur vereinzelt Angaben über die Granulafärbung des pflanzlichen Protoplasmas vor. So z.B. GICKLHORN (1929) erzielte mit verdünnten alkalischen Neutralrotlösungen die im Protoplasma lokaiisierte kristalline Farbstoffspeicherung bei den Blattzellen von Ceratophyllum submersum 
und $C$. demersum. SAKAMURA (1933) bemerkte bei Spirogyra eine Menge von Körnchen im Zytoplasma, die sich mit Neutralrot färben. In den in Teilung begriffenen Sporen von Equisetum arvense treten körnige und fädige Einschlüsse im Zytoplasma in die Erscheinung, die Janusgrün, Dahliaviolett oder Chrysoidin speichern (BECKER und Siemaszko 1936). Neulich bestätigte Sorokin (1938) bei Allium Cepa die Vitalfärbung der Chondriosomen mit Janusgrün B, wie sie schon früher Cowdry (1917), GuILliERMond (1923) usw. auffiel. Chondriosomen lassen sich nach STRUGGER (1938) auch mit Rhodamin B färben, was ich jedoch bei japanischem Material (Zwiebelschuppenepidermis) nicht konstatieren konnte. Bei den Pollenmutterzellen färbt sich das Zytoplasma immer diffus mit einer Anzahl von basischen sowie sauren Farbstoffen. Nur mit Sulfophtaleinen (Bromkresolgrün und Bromkresolpurpur) scheint die granuläre Farbstoffspeicherung aufzutreten, was in geschädigtem Zustand der Zellen immer auffallender wird.

Schließlich möchte ich noch darauf aufmerksam machen, daß eine nicht geringe Anzahl von basischen Farbstoffen weder Zellmembran noch Protoplasma vital färben, so z.B. Janusgrün, Pyronin, Toluylenblau, Neutralrot, Mauvein, Methylenblau, Prune pure, Toluidinblau usw.

\section{Vitalkernfärbung}

Die Vitalfärbung der karyoplasmatischen Strukturen läßt sich wie sonst sehr schwierig erzielen. Chromosomen und Zellkerne haben sich mit folgenden basischen Farbstoffen unverkennbar als vitalfärbbar erwiesen:

Kresylblau, Thionin, Methylviolett, Jodgrün.

Vereinzelt findet man Zellkern und Chromosomen mit Methylgrün und Pyronin schön gefärbt. Vitalität erscheint aber bei solchen Zellen sehr verdächtig. Vitalfärbung der Chromosomen mit sauren Farbstoffen ist mir nur mit Bromkresolgrün gelungen. (YAMAHA 1935, 1937.)

Die erzielte Vitalfärbung der Chromosomen geht bei den Pollenmutterzellen in den meisten Fällen mit diffuser Plasmafärbung einher. Mit Kresylblau lassen sich Chromosomen ziemlich rein färben. Schöne Metachromasie wird zwischen Zytoplasma und Karyoplasma beobachten bei der Vitalfärbung mit Bromkresolgrün und Thionin. Bei Bromkresolgrün beruht sie offenbar auf dem $\mathrm{pH}$-Unterschied (YAMAHA 1935). Mit Thionin färben sich Chromosomen immer bläulich violett (oxydierte Farbe), während das Zytoplasma reduzierte Farbe (schwach rot) annimmt, was auf den rH-Unterschied zwischen Karyo- und Zytoplasma schließen läßt. Halbreduktion des 
Thionins bei pH 7,0 entspricht $\mathrm{rH} \mathrm{15,8.} \mathrm{Ob} \mathrm{das} \mathrm{Zytoplasma} \mathrm{der} \mathrm{Pol-}$ lenmutterzellen Kresylblau und Toluylenblau zu reduzieren vermag, läßt sich nach unseren Ergebnissen der Vitalfärbung nicht ohne Weiteres entscheiden.

Nach Literaturangaben erscheint die Vitalfärbung des Zellkernes im strengen Sinne des Wortes doch auch möglich. Dafür wurden schon eine stattliche Reihe von Farbstoffarten sowohl aus den sauren als auch basischen Gruppen benutzt (vgl. BECKer 1936, S. $461 \mathrm{ff}$.). Thionin und Jodgrün wurden schon früher zur Vitalkernfärbung bei tierischen Zellen verwendet, während Methylviolett nach CAMPBELL (1888), ALBACH (1927) und STRUgGER (1932) auch pflanzlichen Zellkern vital färbt. U̇ber die Vitalfärbung des Zellkernes mit Kresylblau scheint es bisher noch keine Angabe zu geben. Was die Vitalfärbung der Chromosomen betrifft, wurden bislang nur spärliche Beispiele berichtet, und zwar bei Tradescantia, einem klassischen zytologischen Objekt. Dabei kamen Dahliaviolett (CAMPBELL 1888, BECKER 1935), Malachitgrün (BECKER 1935) und angesäuertes Erythrosin (GICKLeHorn 1930) zur Anwendung. Nach Becker (1935, 1936) erscheint die Vitalchromosomenfärbung mit Erythrosin nicht ganz einwandfrei. So scheinen sich Chromosomen nur mit basischen Farbstoffen vital färben zu lassen. Die obengenannten vier Arten der Farbstoffe, wie sie bei den Pollenmutterzellen Chromosomen vital färben, gehören auch zu den basischen. Malachitgrün färben aber hier Chromosomen anscheinend erst postmortal. Ebensowenig konnte ich mit Dahlia und Erythrosin (auch angesäuert bis zu pH 5,6) keine einwandfreie Vitalfärbung der Chromosomen erzielen.

\section{Beeinflussung der Vitalfärbung durch die Ionenkonzen- tration des Mediums (der Farbstofflösung)}

Die Ionenkonzentration der Farbstofflösung beeinflußt auf doppeltem Wege die Ergebnisse der Vitalfärbung, indem sie nicht nur auf die physikochemischen Eigenschaften des Protoplasmas (Quellungsgrad, Ladungsverhältnisse usw.) wirkt, sondern auch den Dissoziationsgrad, die Dispersität usw. der Farbstoffe verändern kann (YAmAHA 1937b). Bei der Vitalfärbung der Pollenmutterzellen erscheint die Ionenkonzentration der Farbstofflösung umsomehr höchst bedeutsam, als sie erfahrungsgemäß für die Gesundheit der betreffenden Zellen entscheidend wirkt. Diesbezügliche Versuche lassen sich aber leider nicht so weitgehend durchzuführen, da die Pollenmutterzellen nur in einem verhältnismäßig engen Bereich der Ionenkonzentration gesund $z u$ leben vermögen. Dabei scheint auch die Ionenart nicht ohne Bedeutung zu sein (YAMAHA u. IshII 1932, YAMAHA 1937). So benutzte ich unten als Lösungsmittel der Farb- 
stoffe ausschließlich 0,1 GM Phosphatpuffer (Gemisch von $\mathrm{KH}_{2} \mathrm{PO}_{4}$ und $\mathrm{K}_{2} \mathrm{HPO}_{4}$ ), worin Pollenmutterzellen am längsten (wenigstens 6 Stunden bei $27^{\circ} \mathrm{C}$ ) gesund zu leben scheinen. Die Pufferlösungen von $\mathrm{pH} 5,7,6,5,7,2$ und 8,0 enthalten je 0,05 oder 0,1 proz. (saure) oder 0,001 proz. (basische) Farbstoffe. Zur Kontrolle wurden weiter auch Farbstofflösungen von derselben Konzentration in umdestilliertem Wasser ausprobiert, deren $\mathrm{pH}$ durch Zusatz von $\mathrm{KOH}$ oder $\mathrm{HCl}$ auf 6,5 gebracht wurde. In diesen Fabstofflösungen leben aber Pollenmutterzellen immer nicht länger als eine Stunde.

Ohne auf die Einzelheiten der Versuchsergebnisse einzugehen, möchte ich mich hier nur auf einige wichtige Schlüsse beschränken. Zunächst ist hervorzuheben, daß der Einfluß der Ionen (H-, K- und Phosphationen) auf die Vitalfärbbarkeit der Protoplasten jenach der Farbstoffart keineswegs ein gleichmäßiger ist. Betreffs sauren Farbstoffes kann man folgendes schließen:

1. Bei denjenigen Farbstoffen, welche Zellmembran rein (d.h. ohne Plasmamitfärbung) färben, wird die Vitalfärbung der Zellmembran offenbar durch H-Ionen bzw. Neutralsalze in der Farbstofflösung begünstigt (so z.B. bei Zyanol extra, Methylblau, Indigkarmin, Anilinblau, Kongorot usw.). Diese Vitalfärbung begünstigende Wirkung der Ionen kommt weiterhin jenach der Farbstoffart in verschiedenen Maßen zur Geltung. Besonders merkwürdig läßt sich diese Ionenwirkung z.B. bei Methylblau und Kongorot bemerken. Im übrigen ist hier nebenbei hervorzuheben, daß Methylblau bei alkalischer Reaktion durch die Pollenmutterzellen leicht reduziert wird.

2. Bei denjenigen sauren Farbstoffen, welche gleichzeitig die Zellwand und das Zytoplasma färben, tritt die Beeinflußung der Vitalfärbung durch die Ionenkonzentration immer nicht deutlich hervor, so z.B. bei Lichtgrün, Bromkresolgrün, Trypanrot usw. Dasselbe gilt auch bei solchen sauren Farbstoffen, wie sie weder Zellmembran noch Zytoplasma färben können (Rubin S, Methylrot, Sulforhodamin usw.)

3. Schließlich tritt die färbungsfördernde Wirkung der Ionen auch da merklich hervor, wo die Vitalfärbung des Zytoplasmas ohne Membranmitfärbung mit sauren Farbstoffen (aus der Gruppe von Nitro-, Azo- und Xanthenfarbstoffen) zu bemerken ist (Aurantia, Tropaeolin OO, Rhodamin B, Eosin, Erythrosin, Bengalrosa usw.). Es sei hier merkwürdig, daß die Vitalfärbung mit Rhodamin B, das sich durch seine hohe Lipoidlöslichkeit auszeichnet, durch Ionenkonzentration nicht unverkennbar beeinflußt wird. Nach STRUGGER (1938) tritt die Vitalfärbung mit diesem Farbstoffe bei Allium Cepa unabhängig von der H-Ionenkonzentration der Farbstoffösung in die Erscheinung, was auch von mir selbst bestätigt wurde. 
Im Zusammenhang hiermit möchte ich hier besonders darauf aufmerksam machen, da $\beta$ im Gegensatz zur Angaben von Drawert (1937), Rhodamin B, ähnlich wie andere saure Farbstoffe, in wässriger Lösung neben H-Ionen Farbstoffanionen abdissozieren kann, was aus der chemischen Konstitution des betreffenden Farbstoffes hervorgeht (vgl. Schultz' Farbstoff-Tabellen Bd. I, 1931, 7. Aufl. S. 365). Nach eigenen Kataphorese-Versuchen ist der Farbstoff zwischen pH 3,5 und 8,0 elektrisch negativ geladen. Erst bei $\mathrm{pH} 2,0$ wandert die Farbe zur Kathode. Rhodamin B verhält sich also vielmehr wie ein saurer Farbstoff als ein amphoterer. Es enthält in seinem Molekül eine freie-COOH-Gruppe. Dementsprechend reagiert die wässrige Lösung von Rhodamin B ziemlich sauer. pH-Messung mittels Antimon- und Glaselektrode ergibt den Wert $\mathrm{pH} 3,7$ bei 0,1 proz. Spezifische Leitfähigkeit derselben Lösung beträgt $0,28 \times 10^{-3}$. welche ungefähr $0,001 \mathrm{n} \mathrm{KCl}$ entspricht.

Was die basischen Farbstoffe anbelangt, so scheinen die Verhältnisse noch verwickelter zu sein. Bei einer Menge von basischen Farbstoffen wird die Vitalfärbung, BETHEscher Theorie gemäß, durch alkalische Reaktion der Farbstofflösung anscheinend begünstigt (Fuchsin, Dahlia, Methylviolett, Methylgrün usw.). Bei einer nicht geringeren Anzahl von basischen Farbstoffen, insbesondere bei den hoch lipoidlöslichen (Nachtblau, Nilbau, Chrysoidin, Neutralrot, Prune pure usw.) läßt sich die Beeinflußung der Vitalfärbung durch die Ionenkonzentration nicht deutlich bemerken. Hier sei merkwürdig, daß die meisten geprüften lipoidlöslichen basischen Farbstoffe die Pollenmutterzellen gar nicht oder nur schwach vital färben können. Bei leicht reduzierbaren Farbstoffen (Thionin, Kresylblau, Methylenblau usw.) wird die Vitalfärbung bei alkalischer Reaktion offenbar gehemmt, was ohne Zweifel auf die Farbstoffreduktion durch das Protoplasma zurückzuführen ist. Der Einfluß des Salzzusatzes zur Farbstofflösung auf die Vitalfärbung mit basischen Farbstoffen erweist sich in den meisten Fällen nicht sehr eindeutig. Bei der Vitalfärbung mit basischen Farbstoffen wirkt das Neutralsalz auf die letzteren bekanntlich blockierend, wodurch die Vitalfärbbarkeit der Protoplasten vermindert erscheint. Diese Wirkung des Neutralsalzes auf die Vitalfärbung macht sich im Allgemeinen bei den Pollenmutterzellen kaum geltend. Die Ursache dafür dürfte man vielleicht in der obengenannten Elektropositivität der Zellmembran der Pollenmutterzellen sehen. Bei einigen basischen Farbstoffen begünstigen die Neutralsalze in der Farbstofflösung Vitalfärbung deutlich. Die Erörterung dieser Frage möchte ich weiterer Mitteilung vorbehalten.

\section{Zusammenfassung}

1. Die Zellmembran der Pollenmutterzellen färbt sich vital mit einer Anzahl von sauren Farbstoffen, aber in der Regel nicht mit den basischen. 
2. Das Zytoplasma ist vitalfärbbar sowohl mit sauren Farbstoffen (Nitro-, Azo- und Xanthenfarbstoffen), als auch mit basischen. Die meisten lipoidlöslichen basischen Farbstoffe färben die lebenden Pollenmutterzellen gar nicht oder nur schwach.

3. Vitalfärbung der Zellkerne und der Chromosomen wird mit einigen basischen Farbstoffen (Kresylblau, Thionin, Jodgrün und Methylviolett) erzielt.

4. Beeinflussung der Vitalfärbung durch die Ionenkonzentration der Farbstofflösung wird zusammenfassend erörtert.

Verfasser spicht an dieser Stelle der „Nippon-Gakuzyutu-Sinkōkwai“-Stiftung für ihre freundliche finanzielle Unterstützung an dieser Arbeit seinen herzlichen Dank aus.

\section{Literaturverzeichnis}

Albach, W. (1927) Zts. f. wiss. Mikr. 44: 333.

Becker, W. A. (1935) Cytologia 6:337.

(1936) Protoplasma 26: 439.

u. Siemaszko, J. H. (1936) La Cellule 45: 29.

Campbell, D. H. (1888) Untersuch. Bot. Inst. Tübingen 2: 569.

Conn, H J. (1936) Biological stains. Geneva N. Y.

Cowdry, N. H. (1917) Amer. J. Anat. 33: 196.

Czaja, A. Th. (1934) Planta 21:531.

(1936) Ebenda 26:90.

Drawert, H. (1937) Flora N. F. 32: 91.

Gicklhorn, J. (1929) Protoplasma $7: 341$.

(1930) Ebenda 10:345.

Guilliermond, A. (1923) C. R. Soc. Biol. Paris 88: 527.

McClung, C. E. and Conn, H. J. (1937) MeClung's Handbook of microscopical technique, 2. Ed. New York S. 573.

Mangin, L. (1892) Bull. soc. bot. de France S. 260.

(1910) C. R. Acad. Sei. Paris 151: 279.

Sakamura, T. (1933) J. Fac. Sci. Hokkaido Imp. Univ. V. 2 : 287.

Sorokin, H. (1938) Amer. J. Bot. 25: 28.

Spek, J. (1937) Ergebn. d. Enzymforsch. 6: 1.

Strugger, S. (1932) Planta 18: 561.

(1938) Protoplasma 30:85.

Yamaha, G. (1920) Bot. Mag. Tokyo 34: (199).

(1935) Cytologia 6: 523.

(1937) Ebenda Fujii-Jubiläumsband S. 617.

(1937b) Bot. Mag. Tokyo 51 : 533.

(1938) Proc. Imp. Acad. Tokyo $14: 81$.

(1938b) Ebenda 14: 83.

u. Ishii, T. (1932) Cytologia $3: 333$.

u. - (1933) Protoplasma 19: 194. 